\title{
Engineering challenges of the JET ITER-like Wall
}

V Riccardo ${ }^{\mathrm{a}}(*)$ on behalf of the ITER-like Wall Engineering Design and Manufacture Team $^{\mathrm{a}-\mathrm{f}}$

${ }^{a}$ Euratom/UKAEA Fusion Association, Culham Science Centre, Abingdon, UK ${ }^{b}$ Ins. für Energieforschung-4, Forschungszentrum Jülich, EURATOM Ass., TEC, Jülich, Germany EFDA-Close Support Unit, Culham Science Centre, Abingdon, UK ${ }^{d}$ Max-Planck-Institut für Plasmaphysik, EURATOM Association, 85748 Garching, Germany 'Association Euratom-CEA, Cadarache, DSM/DRFC, Saint Paul Les Durance, France ${ }^{f}$ Nat. Inst. for Laser, Plasma and Radiation Physics, Ass. Euratom-MEdC, Bucharest, Romania

\begin{abstract}
The ITER-like Wall (ILW) Project offers an integrated test of the ITER reference materials in relevant plasma conditions. The objective of the ILW is to replace all the carbon-based plasma facing components (PFCs) with metal ones: a predominantly beryllium wall and a tungsten divertor. The ILW will have to accommodate enhanced additional heating up to $50 \mathrm{MW}$ for up to 20s, subject to two technical constraints: keep the PFCs inertially cooled only and limit disruption forces on existing infrastructures at the levels set by the present PFCs. In the following it is shown how the ILW can sustain 5MA 4T disruptions, offers a life of over several thousands of high power pulses and can be fully replaced by remote handling. Even if the new wall will not be operational until 2010, the ILW is already making the materials driven operational constraints predicted for ITER a more immediate reality for JET.
\end{abstract}

JNM Keywords: B0100, T1000

PSI Keywords: Beryllium, Divertor, First Wall, Tungsten, Tungsten coated carbon PCAS: 28.52Af, $28.52 \mathrm{Fa}$

(*) JET, Culham Science Centre, Abingdon OX14 3DB, UK - valeria.riccardo@jet.uk 


\section{Introduction}

By installing a beryllium (Be) wall and a tungsten (W) divertor, the ILW project [1] aims at demonstrating that this plasma facing material combination has sufficiently low fuel retention to meet ITER requirements and is compatible with ITER relevant integrated scenarios. The ILW will also show how the interaction among Be, $\mathrm{W}$ and plasma influences plasma performance and PFC life expectancy.

The material layout chosen for the ILW in 2004 is slightly different (and more challenging) than the ITER baseline at the time, which was all-Be main chamber, W brushes in the divertor region and CFC at the target plates. The ILW material combination offers a clean comparison between an all-metal and an all-C JET wall. In addition, it will provide relevant information for the preparation of an all-W divertor ITER option, which has become the favourite layout for the ITER DT phase.

\section{Design of the beryllium main chamber}

The ITER reference design has a nearly continuous actively cooled Be wall surface. The technical constraints for the JET ILW project force this ideal to be compromised. Firstly, an actively cooled design was not feasible given infrastructure limitations. Secondly, the existing JET wall relies on a series of discrete poloidal limiters. The fundamental similarity to ITER is that solid Be is used at the primary contact points between the plasma and the main wall.

The area of greatest compromise to the ideal material combination was imposed by the technical need to maintain or increase the energy handling of the neutral beam (NB) shinethrough regions. This requirement is driven by Advanced Tokamak (AT) scenarios which 
are currently formed at low density and thus high shine-through power, and maintained non-inductively by bootstrap current and NB current drive which is most efficient at low densities and so high shine-through fractions. Although increasing the density of AT scenarios in the run-up to the ILW experiment is a key operational goal, the technical requirement stands. It has been met by employing W-coated CFC tiles of a similar design to the present uncoated CFC ones, some with increased thickness to enhance their thermal inertia. To minimise interactions with the plasma, all main chamber $\mathrm{W}$-coated CFC components are recessed at least $10 \mathrm{~mm}$ behind the limiter trace and in most cases $>25 \mathrm{~mm}$. In situ tests [2] have shown that erosion due to sputtering by the NB shine-through power is not significant, while limiter plasma interaction in the plasma ramp up and down phase is. Other areas of compromise to the ideal material combination are due to a mixture of financial, schedule and manpower constraints. Figure 1 summarises the planned material layout. Only bulk Be PFCs are within $10 \mathrm{~mm}$ of the limiter trace.

\subsection{Electromechanical loads on Be PFCs}

Plasma disruptions produce large changes in magnetic field, which induce eddy currents in conducting materials. The electrical resistivity of $\mathrm{CFC}$ is $\sim 10 \mu \Omega \mathrm{m}$ while that of $\mathrm{Be}$ is only $\sim 0.08 \mu \Omega \mathrm{m}$, so eddy currents go from negligible to dominant load. The eddy currents interact with the local magnetic field to produce a torque, which is strongly dependent on the plate dimensions. Calculations for the main limiter tile types clearly show that a tile of the size of the present CFC tiles would give unacceptable eddy torques, leading to several slicing options being investigated. The chosen design has vertical slices with a large central 
block and 1 to 3 side slices, depending on the toroidal extent of the tile assembly, supported on a carrier via pins. This design, shown in Figure 2, is defined by the balance between conflicting requirements of eddy currents (avoidance of large low-resistance loops) and power handling (minimum number of vertical cuts to be shadowed).

Reserve factors (RF) for the ILW are defined following the ASME specifications [3], and based on avoiding plastic collapse. The working temperature assumed is bulk (not surface) Be parts at $600^{\circ} \mathrm{C}$ and inconel carriers at $400^{\circ} \mathrm{C}$. No fatigue analysis of the electromechanical loads has been carried out as it is not applicable to the ILW: only a small number of pulses is expected to approach the design load, i.e. a disruption occurring with plasma current 6MA and on axis toroidal field 4T. Over a 5-year program, the ILW will see 22500 pulses, of which only $\sim 20 \%$ will be above $3 \mathrm{~T}$ and $3 \mathrm{MA}$. Based on all operations with a divertor, and moving disruptions occurred in the $4.5-5.0 \mathrm{MA}$ bracket to the 5.06.0MA bracket (no disruption $>5 \mathrm{MA}$ has occurred since the divertor structure has been installed), no more than two events will have $100 \%$ of the design load and $<18$ will have $>70 \%$.

Tiles and carriers have been analysed using a combination of first principle calculations and finite element models, both electromagnetic and structural [4]. Nearly all carrier and tile RFs are $>1$. The few carrier/tile RFs $<1$ are in special tiles, i.e. modifications to standard tiles to be compatible with the available space envelope. A large fraction of "gapping" RFs is $<1$. A gapping RF of 1 corresponds to the mating faces of the bolted assembly being on the point of separating when the assembly is loaded by the bolt pre-load and the applied disruption loads. Low gapping RFs are due to low pre-loads (well below the bolt limit), which are needed for remote handling $(\mathrm{RH})$ : to ensure disassembly where experience shows 
that in-vessel operation results in an increase in disassembly torque, typically double the assembly torque. Where gapping can occur, an assessment of the expected bolt stresses shows that several hundreds of cycles can be accommodated before failure.

\subsection{Thermal loads on Be PFCs}

Be melts at $\sim 1278^{\circ} \mathrm{C}$. Starting at $200^{\circ} \mathrm{C}, 6 \mathrm{MW} / \mathrm{m}^{2}$ for $10 \mathrm{~s}$ can be tolerated without melting. However, the tile temperature ratchets while pulsing, up to a hot start of $\sim 400^{\circ} \mathrm{C}$, which will reduce the power handling capacity to $4 \mathrm{MW} / \mathrm{m}^{2}$ for $10 \mathrm{~s}$. Because only limited mechanical properties of $\mathrm{Be}$ are available at elevated temperatures, the strategy adopted was to minimise thermally induced stress as far as practical in order to maximise the fatigue life. The thermal stresses depend on the temperature profile and the degree of constraint in the tiles. The latter has been addressed by using kinematic supports, which allow the tile to both expand and bow. To further relieve local constraints castellations have been introduced and sized following an extensive program of coupled thermo mechanical stress calculations. The low ductility of Be at high temperature could severely limit the fatigue life of an uncastellated tile. With castellations, the surface cyclic strain is eliminated and the limiting stress appears at the colder castellation root. The fatigue life estimate, based on the Coffin relation, is $8300-18000$ cycles depending on the root profile.

Ideally all edges are shadowed. However, all horizontal internal cuts are not shadowed as the ion Larmor radius $\left(\rho_{\mathrm{L}}=0.592 \mu \mathrm{m}\right.$ for $\left.\mathrm{T}_{\text {sep }}=100 \mathrm{eV}, \mathrm{B}_{\mathrm{T}}=3.45 \mathrm{~T}\right)$ is sufficiently larger than the gap width $(350 \mu \mathrm{m})$ to prevent penetration of the ions the grooves. Horizontal gaps between tile assemblies need to be shadowed because much larger (2-3 mm). Shadowing of 
internal vertical edges is impractical from the manufacture point of view and, even if possible, might reduce the overall power handling performance, as it requires locally steeper surfaces (and hence a locally larger power density). The strategy applied is to (0) remove plasma facing assembly and handling holes wherever possible, (1) keep the central block with a shallow toroidal profile and un-shadowed castellations, (2) shadow edges between slices and (3) shadow castellation edges in the side slices. The justification for (1) is that if the surface is sufficiently shallow to offer a small (typically $<40 \mu \mathrm{m}$ ) step to the toroidal direction, even if the power density is very high, the heat sink is sufficient to remove the locally deposited power causing only a minor perturbation to the overall performance of the tile. The plasma facing surfaces of all the Be tile assemblies have been checked for over-heating of exposed edges [5]. A set of plasma limiter scenarios has been investigated in a multi-scale work-program (whole limiter, single tiles and single castellations) taking into account misalignment caused by the manufacturing and the assembly. Comparison of the contributions from radial, poloidal and toroidal face heat loads shows that penetrating field lines only provide $<20 \%$ of the local temperature increase, confirming that the shadowing methodology suits the requirements. By design the limiters are compatible with ohmic limiter phases at start-up and ramp-down and with diverted plasmas with $30 \mathrm{MW}$ loss power (i.e. $45 \mathrm{MW}$ input and $30 \%$ radiation) for $20 \mathrm{~s}$ with a clearance of 3 scrape-off-layer lengths, which is much smaller than typical clearances of $3-5 \mathrm{~cm}$. However, accidental wall contact of a plasma at the full heating power would lead to localized melting in $<140 \mathrm{~ms}$. 


\subsection{Further boundary conditions to the Be design}

2.3.1 Ion cyclotron resonance heating (ICRH) image currents: In the vicinity of the ICRH antennas image currents are driven on conductive structures. The ICRH antennas are on the outer wall of the vessel and have poloidal limiters to their sides, and private protections inside, above and below them. The poloidal limiters will be screened from image currents by interposing flux excluding rails, same concept and location as presently, but different design to be compatible with the new carriers. However, there is not enough space to deflect the flux from the private protection tiles, so the attachments between the Be tiles and their inconel carriers have been made compatible with the heating due to the induced currents by using $\mathrm{Cu}$ plated disk springs.

2.3.2 RH compatibility: In addition to the requirement for gripping features, $\mathrm{RH}$ sets two parameters critical to the Be tile design: (1) the minimum gap between tile assemblies and (2) the maximum weight of the payload ( $\sim 10 \mathrm{~kg}$ in the main chamber) and therefore the vertical extent of the limiter tiles. The combination of minimum gap and maximum height has driven the design of the plasma facing surface: because horizontal cuts between tile assemblies need to be shadowed, poloidally higher tiles are more efficient.

\section{Design of the tungsten divertor}

One of the eight rows of tiles in the divertor will be made of bulk W, while the others of Wcoated CFC. The bulk W row, located where the outer strike point is planned to be most often, combined with the power upgrade, offers the opportunity to investigate melt layer formation and stability, along with the impact on the subsequent plasma operation, in the later phase of the ILW exploitation. In additional to the scientific merits, this is also part of 
a strategy to minimise the risk of damaging the divertor. If W-coatings were to melt surface tension would lead to beads, whereas melt layers on bulk material is expected to show a much more benign behaviour.

\subsection{Design of the $W$-coated CFC divertor}

On the basis of $\mathrm{W}$-coating $\mathrm{R} \& \mathrm{D}, 200 \mu \mathrm{m}$ vacuum plasma spray (VPS) was originally chosen. However, this reference has been reviewed in light of further pre-production tests and analysis of W coatings exposed in JET [6], resulting in physical vapour deposition (PVD) W/Re multi-layer, of thickness between $14 \mu \mathrm{m}$ and $30 \mu \mathrm{m}$ (to be finalised following tests), being selected. The decision to proceed with a thinner coating could reduce the life of the W-coated tiles due to erosion during operation: coated tiles exposed for a few years operation at a time in JET show on average $0.01-0.06 \mathrm{~nm} / \mathrm{s}$ [6] are lost due to physical sputtering. However erosion amplification, a factor of 4, due to the roughness of the surface has been observed on JET samples. Therefore $14 \mu \mathrm{m}$ are expected to survive $\sim 60000 \mathrm{~s}$.

The tests carried out in the R\&D also indicate that the maximum temperature of the $\mathrm{W}$ coated CFC surface should be kept $<1600^{\circ} \mathrm{C}$ [7], to limit the generation of defects due to the acceleration of the formation of a brittle interlayer of $\mathrm{W}$ carbide. The birth of cracks and blisters is enhanced by the mismatch between the CFC and the $\mathrm{W}$ coefficients of thermal expansion. Despite knowing the grade of CFC used in the JET divertor is less suited for Wcoatings than other grades or fine grain graphite, it was decided to keep the divertor material unchanged, because limited design resources did not allow the divertor assembly to be redesigned to become compatible with a different C-based material and limited R\&D 
resources could only address the selection of the coatings rather than the combined selection of coatings and substrates.

The divertor tiles are subject to near steady state and transient loads, mainly ELMs and disruptions. Both loads need to fit within the coating temperature limits. ELM-like tests [8] show that $14 \mu \mathrm{m}$ PVD coatings can tolerate at least 1000 cycles started near room temperature with $330 \mathrm{MW} / \mathrm{m}^{2}$ applied for $1 \mathrm{~ms}$, this energy density is smaller but comparable with the one observed [9] in experiments aimed at producing extreme ELMs in JET. Consequently most JET ELMs should be tolerated by the W-coatings, but infrequent high energy ELMs might not and require specific protections to be implemented.

\subsection{Design of the bulk $W$ divertor}

There will be 48 bulk W Load Bearing Septum Replacement Plates (LBSRPs) [10] assemblies in the divertor. A module, Figure 3, is made of two tile assemblies, each includes adaptor plate, wedge, eight stacks of W lamellae, besides two small W-coated CFC protections and various fixtures and fittings. In order to meet the strict requirements on suppression of eddy currents, the inconel wedge has a tree-like structure. Each W stack is made of 24 lamellae with $1 \mathrm{~mm}$ gaps between them. TZM spacers fit between the lamellae, every other spacer is electrically insulated to minimise eddy current loops. Each stack of lamellae and spacers is attached to the wedge with a chain pulled down by springloaded bolts, with the design aiming at keeping all refractory metals in compression, as JET operational temperature excursions can cross the ductile-to-brittle transition temperature. 
The weight of the assembly, set by the RH payload in the divertor, determines the amount of $\mathrm{W}$, and thus its thermal inertia. $\mathrm{RH}$ sets also the gap between the tile assemblies and hence the stack chamfer angle. In addition, each lamella is profiled to ensure internal edges are not exposed. The combined effect of the two chamfers produces a power density amplification factor with respect to a continuous conical annulus between 2.4 (steep, $4^{\circ}$, field line angles) and 5.4 (shallow, $1^{\circ}$, field line angles), compared to respectively 1.8 and 3.2 if only the long-range chamfer is required.

\section{Impact on operations}

Current tokamaks, including JET, which have inertially cooled C-based PFCs and relatively low stored energy compared to ITER, enjoy a situation where the PFCs are almost indestructible because the $\mathrm{C}$-bloom is a relatively benign thermal limit which at most rounds off the offending edges. This will no longer be true with a Be wall and a $\mathrm{W}$ divertor. It is therefore necessary to develop techniques to minimise the risk of unscheduled melting, such as high steady state power density, NB shine-through power, hot spots associated with hot ions or electrons, transient power loads from ELMs and disruptions.

To maximise machine performance and approach more closely ITER conditions, JET will have to make full use of the heating (40-50MW) and plasma current (5-6MA) capability. This will mean operating well beyond the point at which, if control is lost, serious damage can result. This risk can be minimised by phasing the programme, by making more extensive use of control systems and protections and by avoiding known incompatible programmes. Protections need to be fail-safe, robust and within the boundaries of the engineering limits. These characteristics often make the protections inflexible and severely 
constrain operation. Therefore limit avoidance control schemes could become increasingly important. For example the use of NB with low density plasmas will benefit from a control system able to switch off only the beams with their footprint surface temperature above a critical value, substitute them with suitable others and allow them to be switched back on once the footprint has cooled sufficiently. The energy deposited in each region of the wall could be estimated in real time to instigate a high clearance, low power, disruption-free soft stop when a critical value is reached. In JET this type of control has to be based on estimates of wall temperatures, with only a few of them validated against measurements. Consequently, even this optimised way of using the operating space will suffer from some conservatism (pessimistic assumptions on what cannot be validated or measured). In order to support the development and the validation of limit avoidance control systems and protections, the ILW includes the installation of several arrays of main chamber thermocouples. In addition, the ILW supported the modification of the shutter of the present wide angle Infra-Red (IR) camera to view the NB shine-through region and validate models. Moreover, the ILW supports the installation of a second main chamber IR camera viewing the Lower Hybrid launcher. If the IR cameras prove more routinely available and overall reliable than presently, these could be integrated in protection systems allowing wider operational space. However, a priori prediction of disruptions as well as of the approaching of operating regions where infrequent ELMs are likely should be developed and combined with algorithms capable of steering the plasma towards ILW-compatible conditions.

\section{Conclusions}


Within a tight, externally imposed, schedule the design of the ILW had to provide the full replacement of a C-based set of PFCs with metal-based one, whilst keeping PFCs inertially cooled and compatible with the existing in-vessel infrastructure. Some critical material choices might have been different if more time for pre-contract R\&D had been available. Notwithstanding this, the main technical objectives of the design have been achieved and it is now for the exploitation to fit within the new limits set by the ILW, which are generally in line with those expected at the project set-up and represent a gentle introduction to the materials driven operational constraints predicted for ITER.

\section{Acknowledgements}

This work, jointly funded by EPSRC via the JET Operating Contract and the European Communities under the contract of Association between EURATOM and UKAEA, was carried out within the framework of the European Fusion Development Agreement. The views and opinions expressed herein do not necessarily reflect those of the European Commission.

\section{References}

[1] J. Pamela e al., J. Nucl. Mater. 363-365 (2007) 1.

[2] P. Coad et al., J. Nucl. Mater. these Proceedings.

[3] ASME Section VIII. Unfired Fusion Welded Pressure Vessels.

[4] V. Thompson et al., Fusion Eng. Design 82 (2007) 1706.

[5] M. Firdhaouss et al., J. Nucl. Mater. these Proceedings.

[6] G. Matthews et al., J. Nucl. Mater. these Proceedings. 
[7] H. Maier et al., J. Nucl. Mater. 363-365 (2007) 1246.

[8] T. Hirai, JET-EFDA report, JW6-FT-3.35 to be submitted

[9] R. Pitts et al., J. Nucl. Mater. these Proceedings.

[10] P. Mertens et al., J. Nucl. Mater. these Proceedings. 


\section{Figure captions}

Figure 1 - View of the present JET main chamber with indications of how the CFC tiles will be replaced.

Figure 2 - Inner Wall Guard Limiter tile (exploded view, top, and prototype, bottom). The 5 castellated Be slices have inter-slice and outer slice internal toroidal edges ski-slope shadowed. The slices are held on an inconel carrier by pins which allow bowing under thermal load. The RH bolts are designed to be shadowed by the next installed tile.

Figure 3 - Bulk W LBSRP module. 


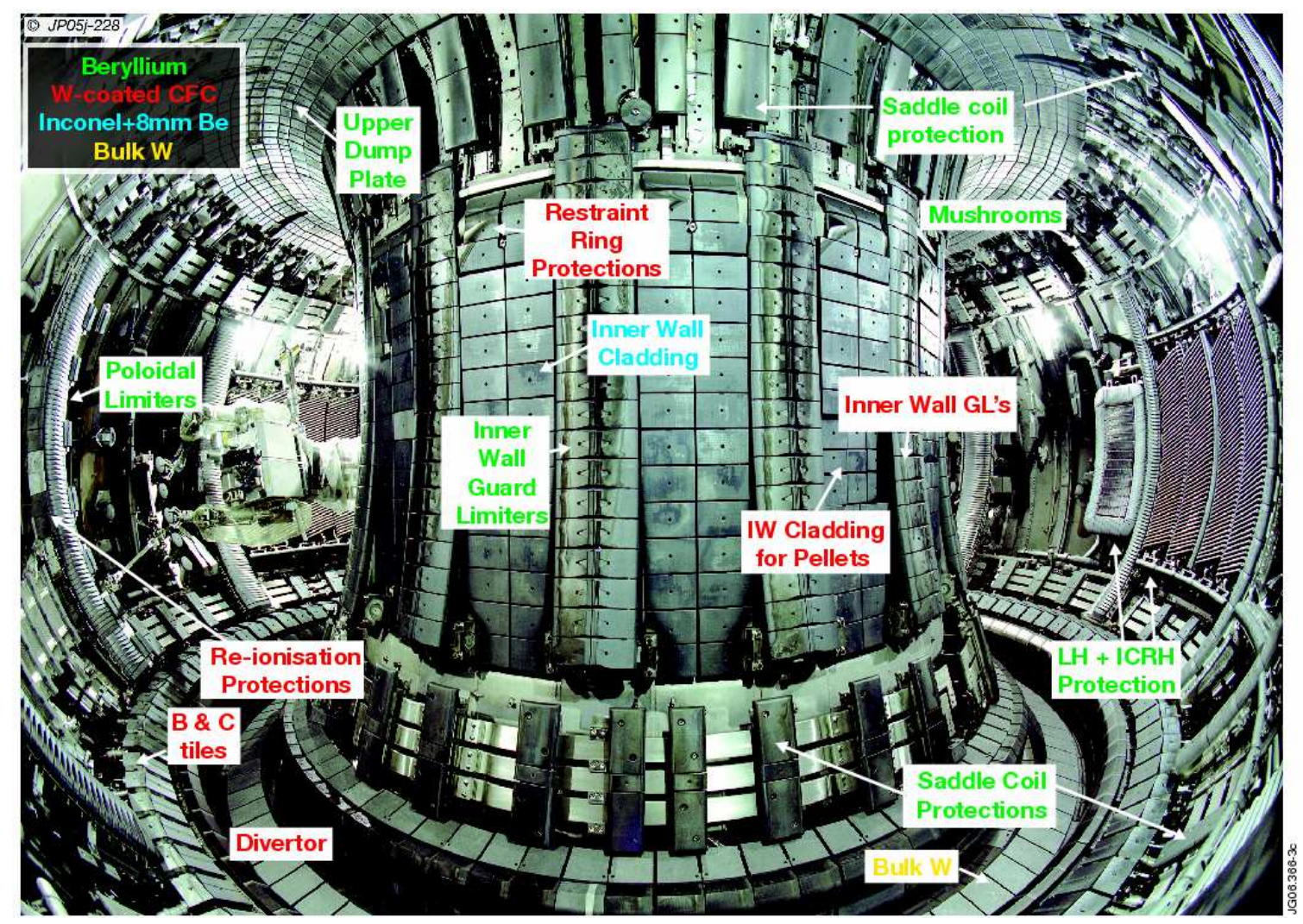

Figure 1 

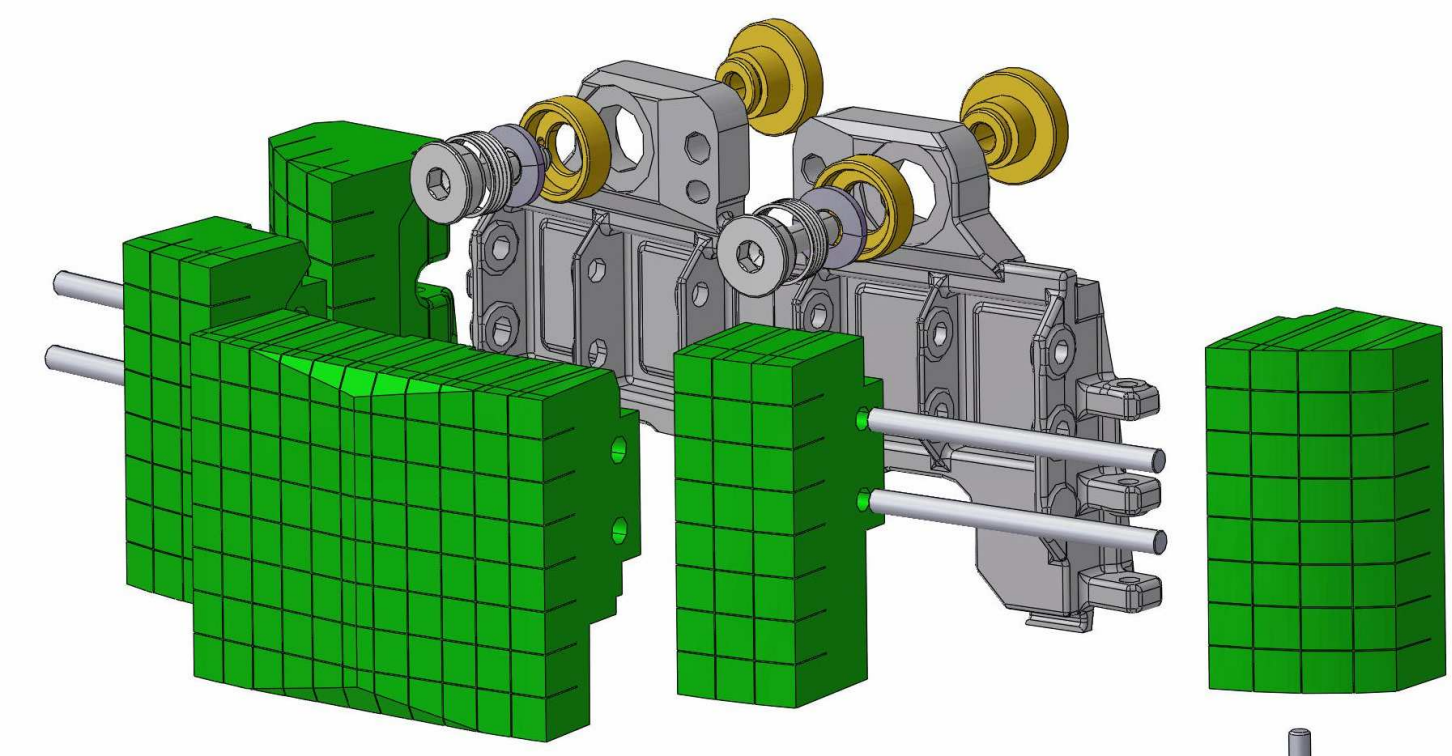

1

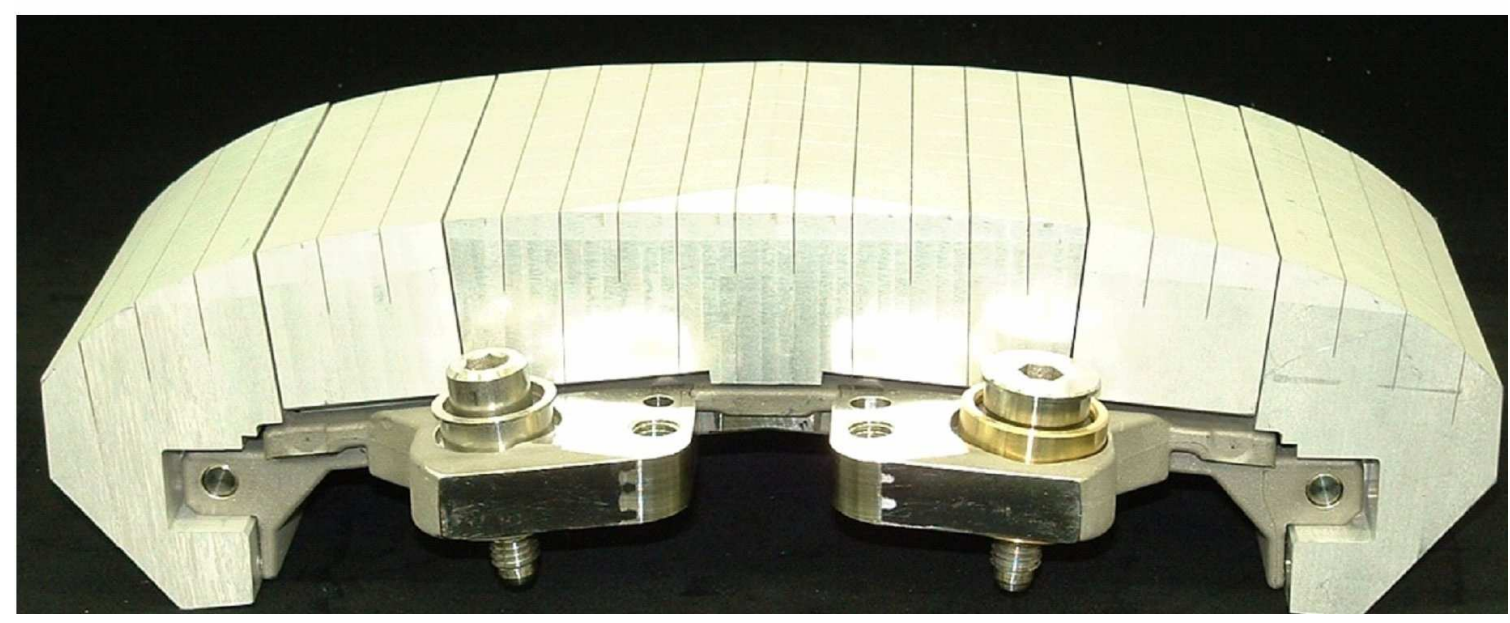

Figure 2 


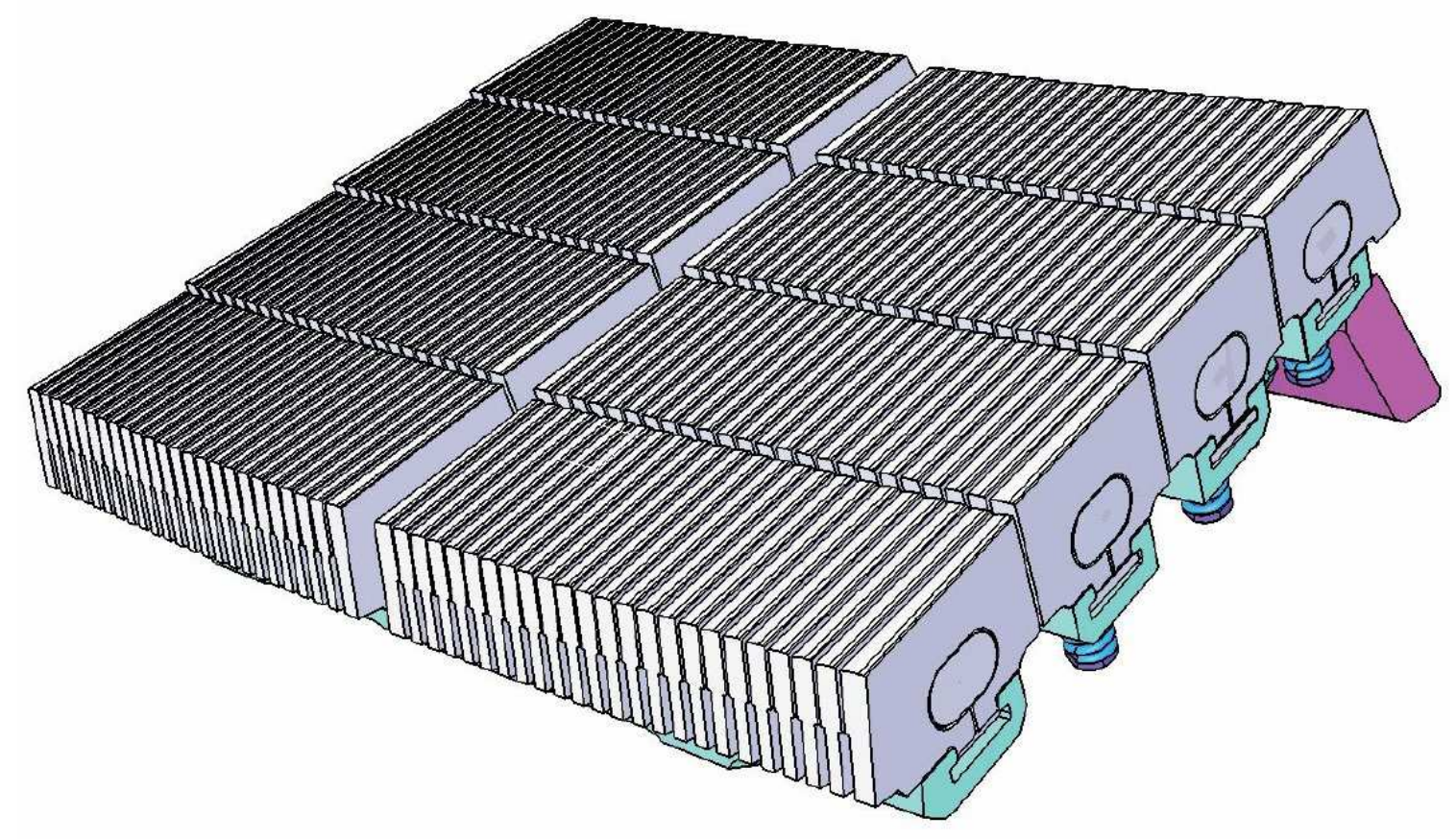

Figure 3 